\title{
Scaling theory for the free-energy barrier to homogeneous nucleation of a non-critical phase near a critical point
}

\author{
Richard P. Sear \\ Department of Physics, University of Surrey \\ Guildford, Surrey GU2 7XH, United Kingdom \\ email: r.sear@surrey.ac.uk
}

\begin{abstract}
Homogeneous nucleation of a new phase near an Ising-like critical point of another phase transition is studied. A scaling analysis shows that the free energy barrier to nucleation contains a singular term with the same scaling as the order parameter associated with the critical point. The total magnetisation of the nucleus scales as the response function and so it diverges. Vapour-liquid critical points are in the Ising universality class and so our results imply that near such a critical point the number of molecules in a nucleus of a another phase, such as a crystalline phase, diverges as the isothermal compressibility. The case where symmetry prevents coupling between the nucleus and the order parameter is also considered.
\end{abstract}

\section{INTRODUCTION}

It is rare for a condensed matter system to possess only one phase transition, which raises the question of how neighbouring phase transitions affect each other. There are many possible effects, and here we consider one of them, namely how nucleation associated with one phase transition is affected by the critical point of a second transition. Consider a first-order phase transition, transition $\alpha$, which occurs at a temperature $T_{\alpha}$. Above $T_{\alpha}$ the high-temperature phase, call it $\alpha_{H T}$, is the equilibrium phase while below it the low-temperature phase, $\alpha_{L T}$, is the equilibrium phase. Fig. [1 is a schematic of the temperature axis of the phase diagram. If we cool the hightemperature phase below $T_{\alpha}$ then it ceases to be the equilibrium phase. At some degree of supercooling a nucleus of the low temperature phase will appear and grow into a macroscopic $\alpha_{L T}$ phase. The initial step, the formation of a nucleus of the $\alpha_{L T}$ phase, is an activated process a free energy barrier must be surmounted [1]. The rate at which nuclei overcome a barrier of height $\Delta F^{*}$ scales as $\exp \left(-\Delta F^{*} / k T\right)$ and so this rate is very sensitive to the barrier height [2]. Here we calculate $\Delta F^{*}$ for nucleation near a critical point of another phase transition, which we call transition $\beta$. Transition $\beta$ is Ising-like. So, we have two phase transitions: a strongly first order phase transition $\alpha$, whose dynamics we are studying, and a transition with a critical point, transition $\beta$, which lies at a temperature $T_{\beta}<T_{\alpha}$ and so occurs within the $\alpha_{H T}$ phase when it is not the true equilibrium phase. At an Ising critical point the thermodynamic functions contain singular power-law terms with exponents which depend only on dimensionality 3, 1 . Below, we show that $\Delta F^{*}$ also contains a singular power-law term with exponents which depend only on dimensionality. The singular part of $\Delta F^{*}$ has the same form as that in the order parameter, so for example, it scales with temperature as $\left|T-T_{\beta}\right|^{\beta}$, where $\beta$ is the usual critical exponent [3, 4. This singu- lar part means that the derivative of $\Delta F^{*}$ with respect to temperature diverges as the critical point is approached: the barrier to nucleation is very sensitive to changes in temperature near the critical point. Which is true for any system with an Ising-like critical point.

The scaling analysis here follows on from mean-field calculations performed by the author 5,6 , see also Ref. [7]. Which in turn followed on from the pioneering simulations of ten Wolde and Frenkel [\$] who were the first to appreciate that critical fluctuations could reduce $\Delta F^{*}$ near the critical point of a metastable fluid-fluid transition. The results of the mean-field calculations are consistent with the scaling analysis here; if we take the equations of the present work and set the exponents to their (incorrect) mean-field values we reproduce the exponents found in Refs. [5, 6]. Finally, see the work of Dixit and Zukoski [9] for an alternative approach to nucleation near a critical point; one where dynamics are explicitly but approximately included.

Perhaps the easiest systems in which to observe the effects found here, are mixtures of liquids. There nucleation of either the vapour phase or a crystalline phase may occur near the continuation of a line of critical solution temperatures, i.e., near to a critical point of liquidliquid demixing. A line of critical solution temperatures does not stop when the liquid ceases to be the equilibrium phase but continues into the region where it is metastable with respect to a transition to a vapour or crystalline phase. It is this continuation that affects nucleation.

The study of nucleation near a critical point was sparked by interest in the nucleation of the crystalline phase of globular proteins 10. At least some globular proteins have a fluid-fluid critical point within the fluid-crystal coexistence region [11, 12, The fact that the predictions made here are universal is very useful with regard to protein crystallisation as the interactions between protein molecules are generally rather poorly understood, particularly before they have been crystallised 
and their structure determined [13. We know that our predictions will apply to nucleation near any fluid-fluid critical point found in a protein solution, without needing to know anything about the protein-protein interactions.

\section{THEORY FOR NUCLEI WHICH COUPLE TO THE ORDER PARAMETER}

We will describe the critical point of transition $\beta$ using the standard language of Ising spin systems, although of course our results also apply to critical points in fluids. The order parameter is $m$, the temperature $t=\left(T-T_{\beta}\right) / T_{\beta}$, and the field is $h$ and couples to the order parameter as $-h m$. The critical point is then at $t=0, h=0$, see Fig. 1. For fluid systems the external field $h$ is the chemical potential minus that at coexistence, and $m$ is a density difference. We set the lattice spacing to $a$. For definiteness we set $m$ to be positive in the nucleus. The symmetry between $+m$ and $-m$ means we can do so without loss of generality.

The dynamics of a first-order phase transition starts with nucleation [2, 田, where a microscopic nucleus of the new phase first appears. The nucleus at the top of the barrier, with the highest free energy $\Delta F^{*}$, determines the rate and so we will consider only this nucleus. It is generally called the critical nucleus. Note that conventionally the word critical is used to denote both a continuous transition and the nucleus at the top of the barrier although there is no connection between the two uses of the word. Although this terminology is a little unfortunate we will use it here and so we will be studying a critical nucleus near a critical point.

For nucleation at a strongly first-order phase transition (which transition $\alpha$ is assumed to be), the nucleus must contain a compact core of spins near their state in the bulk $\alpha_{L T}$ phase, as only in this state do they have a lower free energy than in the $\alpha_{H T}$ phase. For all strongly firstorder transitions, the free energy will be higher for spins intermediate between the two phases, here $\alpha_{H T}$ and $\alpha_{L T}$. It is this core which will grow to form the $\alpha_{L T}$ phase. See for example Refs. [2, 4] which discuss the classical theory for nucleation which is based on this observation.

Now, this core of spins will perturb the surrounding spins out to a distance of the correlation length $\xi$. Thus, the volume perturbed is of order $\xi^{d}$ and so diverges as the critical point is approached. $d$ is the dimensionality. The volume around the core where $m$ is small we term the fringe. As in our earlier mean-field calculations [5, 6] we split the nucleus into a core and a fringe. This is illustrated schematically in Fig. 2. As $\alpha$ is a strongly first-order transition, the order parameter of transition $\beta$, $m$, in the core will be of order unity, i.e., not small, and therefore far above the values of $m$ reached by critical fluctuations. The critical fluctuations will however affect the fringe where $m$ is small. We note that the compact core of spins resembles a small colloidal particle in that it is a compact, roughly spherical object, which interacts with and thus perturbs its surroundings. The problem of a colloidal particle in a near critical system has been considered, see Refs. 14 17.

The core of the nucleus will be much smaller than the correlation length $\xi$ near the critical point as it will be only a few lattice sites across whereas $\xi$ diverges [18]. Thus, near the critical point, the core is a point-like perturbation which couples to the order parameter $m$. It will couple to $m$ unless this is prevented by a symmetry. Point-like perturbations near a critical point are generic - all point-like perturbations to the order parameter $m$ perturb large length-scale critical fluctuations in the same way, differing only in a scale factor. Near the critical point where $\xi \gg a$, scale invariance implies that any perturbation of size much less than $\xi$ must cause a order parameter perturbation of the same form, because all such perturbations are simply interrelated by a change of length-scale. The presence of a nucleus at the origin $\mathbf{0}$ must therefore produce (at large distances) the generic perturbation to $m$. We define the thermal average excess of $m(\mathbf{r})$ due to the presence of a nucleus at the origin as

$$
<m_{n}(\mathbf{r})>=<m(\mathbf{r})>_{n}-<m(\mathbf{r})>,
$$

where $<m(\mathbf{r})>_{n}(<m(\mathbf{r})>)$ is the thermal average value of $m(\mathbf{r})$ when there is (is not) a nucleus at the origin. Compare this to the 2-point correlation function, $G(\mathbf{r})$, for the order parameter, defined by

$$
G(\mathbf{r})=<m(\mathbf{0}) m(\mathbf{r})>-<m(\mathbf{0})><m(\mathbf{r})>
$$

Note that $G(\mathbf{r})$ is the thermal average of $m(\mathbf{r})$ if $m(\mathbf{0})$ is fixed to 1. As a point perturbation is generic for the fluctuations on length scales $\gg a$, the function $\left\langle m_{n}(\mathbf{r})\right\rangle$ can only differ from $G(\mathbf{r})$ by a scale factor when $r \gg a$. Calling this scale factor $C$,

$$
<m_{n}(\mathbf{r})>=C G(\mathbf{r}) \quad r \gg a .
$$

$C$ will depend on the properties of the core. This result has been found before not for a nucleus but for a small colloidal particle immersed in a near-critical fluid [14 17. Defining the total excess of $m$ due to the presence of the critical nucleus as $m^{*}$, we have

$$
m^{*}=\int \mathrm{d} \mathbf{r}<m_{n}(\mathbf{r})>
$$

Splitting the integration at $r_{c}$ which satisfies $a \ll r_{c} \ll \xi$, and using Eq. (3) for the integrand of the integral for $r>r_{c}$ we have

$$
m^{*}=\int_{r<r_{c}} \mathrm{~d} \mathbf{r}<m_{n}(\mathbf{r})>+C \int_{r>r_{c}} \mathrm{~d} \mathbf{r} G(\mathbf{r}) .
$$


Now, the response function $\chi$ is equal to the integral over $G(\mathbf{r})$ [3, 团

$$
\int \mathrm{d} \mathbf{r} G(\mathbf{r})=\chi,
$$

and it contains a leading order singular part $\chi_{S}$ coming from fluctuations on a length-scale of the correlation length $\xi$, and so coming from those parts of the integral where $r=O(\xi)$. Thus the second integral of Eq. (5) for $m^{*}$ contains $\chi_{S}: m^{*}$ is singular at the critical point. Near the critical point $\chi_{S}$ dominates $\chi$ and so the term $C \chi_{S}$ in the second integral of Eq. (5) will dominate $m^{*}$. Thus, we have for the leading order singularity in $m^{*}, m_{S}^{*}$,

$$
m_{S}^{*}=C \chi_{S}=C|t|^{-\gamma} X_{ \pm}\left(h /|t|^{\beta \delta}\right),
$$

where $X_{ \pm}$are scaling functions, and $\gamma, \beta$ and $\delta$ are critical exponents. $X_{+}$is the scaling function above the transition, $t \geq 0$, while $X_{-}$is the scaling function below the transition, $t \leq 0$. The second equality is obtained by substituting the standard scaling expression for $\chi_{S}$, 3, , 1 . Close to the critical point, the total order parameter excess associated with the nucleus, $m^{*}$, diverges with the precisely the same scaling behaviour as the order parameter response function, $\chi$.

Having determined the size of the nucleus, we wish to know its free energy. We start by splitting the free energy barrier into a nonsingular part $\Delta F_{N S}$ plus a leading order singular part $\Delta F^{*}$,

$$
\Delta F^{*}=\Delta F_{N S}^{*}+\Delta F_{S}^{*}
$$

The leading order singular part, $\Delta F_{S}^{*}$, of $\Delta F^{*}$, is expected to have the usual scaling form, of a power of $|t|$ times a function of $h /|t|^{\beta \delta}$,

$$
\Delta F_{S}^{*}=|t|^{x} Y_{ \pm}\left(h /|t|^{\beta \delta}\right),
$$

with $x$ an as yet unknown exponent. To determine $x$ we note that the derivative of the free energy of the system $F$ with respect to $h$ is,

$$
\frac{\partial F}{\partial h}=-M,
$$

where $M$ is the total order parameter $m$ of the system. Thus, as $\Delta F^{*}$ is the free energy with a nucleus at the origin minus that without a nucleus at the origin, then the derivative of $\Delta F^{*}$ with respect to $h$ is simply the negative of the total order parameter with a nucleus minus that without a nucleus [19],

$$
\frac{\partial \Delta F^{*}}{\partial h}=-m^{*}
$$

This is for the complete $\Delta F^{*}$. The leading-order singular term in $\Delta F^{*}$, denoted by $\Delta F_{S}^{*}$, will yield minus the leading-order singular term in $m^{*}$, upon differentiation with respect to $h$

$$
\frac{\partial \Delta F_{S}^{*}}{\partial h}=-m_{S}^{*}
$$

The derivative with respect to $h$ of scaling functions like $Y_{ \pm}\left(h /|t|^{\beta \delta}\right)$, brings an additional $|t|^{-\beta \delta}$ factor. Thus for Eqs. (9) and (12) to yield the correct exponent $-\gamma$ for $m^{*}$ we require that $x-\beta \delta=-\gamma$. Using the relation between exponents $\beta \delta=\beta+\gamma$ [3, 迆 we obtain $x=\beta$. So, setting $x=\beta$ in Eq. (9) we obtain

$$
\Delta F_{S}^{*}=|t|^{\beta} Y_{ \pm}\left(h /|t|^{\beta \delta}\right),
$$

the singular part of the free energy scales with the same exponent, $\beta$, as the bulk order parameter. This is for Ising-like systems but we expect that analogous singular terms will be found near critical points of transitions with order parameters which are not scalars, for example in the Heisenberg model where the order parameter is a three-dimensional vector.

As $\beta>0, \Delta F_{S}^{*}=0$ at the critical point, and so the universal singular part is zero. The free energy barrier $\Delta F^{*}$ at the critical point is equal to some non-universal value. However, as $\beta<1$, the derivative of $\Delta F^{*}$ with respect to temperature diverges as $|t|^{\beta-1}$ as the critical point is approached at $h=0$. The signs of the divergences as the critical point is approached from above and below are those of $Y_{ \pm}(0)$ which are unknown. However, on physical grounds we might expect critical fluctuations to reduce the barrier to nucleation, and this is what is found in the mean-field theory calculations for $T>T_{\beta}$ of Ref. [6], i.e., within mean-field theory it has been found that $Y_{+}(0)>0$. If $Y_{ \pm}(0)$ are both positive, then at fixed $h=0$, the free energy barrier will have a local minimum at $T=T_{\beta}$. As the derivative of $\Delta F_{S}^{*}$ diverges, near the critical point the variation in $\Delta F^{*}$ is dominated by that in $\Delta F_{S}^{*}$, and so if $\Delta F_{S}^{*}$ has a minimum at the critical point so does $\Delta F^{*}$.

Along the critical isotherm,

$$
\Delta F_{S}^{*}=\Sigma \operatorname{sgn}(h)|h|^{1 / \delta} \quad t=0,
$$

where $\Sigma$ is an amplitude and the scaling with $h$ is fixed by the requirement that it be the same as that of $m$. From Eq. (12) we see that as $m_{S}^{*}>0$ then the $h$ derivative of $\Delta \overrightarrow{F_{S}^{*}}$ must be negative. For this to be true we must have that $\Sigma<0$. Note that $m_{S}^{*}>0$ because we set $m$ in the core to be $>0$, if the nucleating phase has instead $m<0$, then in that case $\Sigma>0 . \Sigma$ is a function not only of the properties of the critical system but of the nucleating phase. At the critical point the rate of decrease of $\Delta F^{*}\left(=m^{*}\right)$ diverges, so near the critical point the nucleation barrier is a rapidly decreasing function of $h$. 


\section{A. Critical amplitude ratios}

In the $h=0$ limit, the scaling functions $X_{ \pm}$of $\chi_{S}$, Eq. (7), simplify

$$
\chi_{S}=\Gamma_{ \pm}|t|^{-\gamma} \quad h=0
$$

where $\Gamma_{ \pm}=X_{ \pm}(0) . \quad \Gamma_{ \pm}$are critical amplitudes, and are non-universal. However, the critical amplitude ratio $\Gamma_{+} / \Gamma_{-}$is universal [4, 20]. The fact that not only the exponents but the critical amplitude ratios are universal means that we can even make a universal prediction about the ratio of the size of the nucleus just above the critical point to that just below. If we take the ratio $m_{S}^{*}(+|t|) / m_{S}^{*}(-|t|)$, we see, using Eq. (15), that

$$
\frac{m_{S}^{*}(+|t|)}{m_{S}^{*}(-|t|)}=\frac{\Gamma_{+}}{\Gamma_{-}} \quad h=0,
$$

which is universal. As near the critical point $m_{S}^{*}$ dominates $m^{*}$, we have that near the critical point: 1) the excess magnetisation associated with the nucleus is the same in each of the two coexisting phases at some $t<0$, and 2) that the ratio of $m^{*}$ at $|t|$ to the common value of the excess $m$ in the coexisting phases at $-|t|$ is universal. In three dimensions $\Gamma_{+} / \Gamma_{-}=4.9$ [4,20] so the nucleus is approximately five times bigger $|t|$ above the transition than $-|t|$ below. Via the nucleation theorem 21 24 it is possible to estimate the size of the critical nucleus. Thus measurements on nucleation rates can potentially be used to estimate not only critical exponents but critical amplitude ratios.

\section{DYNAMIC SCALING FOR THE EQUILIBRATION TIME NEAR $T_{\beta}$}

Now, the singular part of the barrier, $\Delta F_{S}^{*}$, is universal because it originates in long wavelength fluctuations, fluctuations with wavelengths $\gg a$, where the microscopic details of the system are irrelevant. However, these long wavelength fluctuations are slow, firstly as a direct consequence of them having a long wavelength (this applies if $m$ is a conserved variable 4 ), and secondly because as the critical point is approached the free energy becomes a very flat function of the order parameter which reduces the driving force which relaxes fluctuations. Dynamic scaling theory yields for the characteristic relaxation time $\tau_{R}$ ( $=2 \pi$ over the characteristic frequency) of a small wavevector mode, $k<<a^{-1}$, near a critical point 4. 25

$$
\tau_{R}=k^{-z} D_{ \pm}\left(k \xi, h /|t|^{\beta \delta}\right),
$$

the relaxation time of a mode of wavevector $k$ at temperature $t$ and field $h$. The exponent $z$ is a dynamic scaling exponent. Dynamic scaling exponents depend not only on the symmetry of the order parameter and the dimensionality as the static exponents, $\beta, \gamma$, etc. do, but also on whether the order parameter $m$ is conserved or not. If $m$ is the magnetisation then it can vary locally without transport of $m$ and so it is non-conserved, but if $m$ is a density difference then as the total number of molecules is conserved it cannot vary in a volume of space without transport in or out of that volume, so it is then a conserved variable. In either case $z$ is positive. Assuming that fluctuations with wavelengths larger than the correlation length can be ignored we have that at a given $t$ and $h$, the longest relevant relaxation time is that of a mode of wavevector $k=2 \pi / \xi$. Calling the relaxation time of this mode $\tau_{\xi}$ we have, from Eq. (17),

$$
\begin{aligned}
& \tau_{\xi}=\xi^{z}(2 \pi)^{-z} D_{ \pm}(2 \pi, 0) \\
& \tau_{\xi}=\tau_{ \pm}|t|^{-z \nu} \quad h=0,
\end{aligned}
$$

where we have set $h=0$ for simplicity, the generalisation to non-zero fields is easy. The second line used the scaling for the correlation length $\xi=\xi_{ \pm}|t|^{-\nu}$ for $h=0$, and the amplitudes $\tau_{ \pm}=\xi_{ \pm}^{z}(2 \pi)^{-z} D_{ \pm}(2 \pi, 0)$.

Our expression for the free energy barrier to nucleation, Eq. (8), includes a singular part $\Delta F_{S}^{*}$ coming from long-wavelength fluctuations. This singular part is an equilibrium quantity: it is the free energy change of equilibrium fluctuations due to the nucleus. Note that here by equilibrium we mean that the fluctuations in $m$ etc. have relaxed to equilibrium, the system is not at true equilibrium as it is in the $\alpha_{H T}$ phase below $T_{\alpha}$. Thus, our expression for the free energy barrier, Eq. (8), is only meaningful once a time much larger than $\tau_{\xi}$ has elapsed at that temperature and so the fluctuations in $m$ have equilibrated.

However, $\tau_{\xi}$ diverges as the critical point is approached so the nearer we are to the critical point the longer we have to wait before Eq. (8) gives the correct barrier to nucleation. For $m$ conserved, $z=4-\eta$ and so putting in the values for $\eta$ and $\nu$ we have that $\tau_{\xi}$ scales as $|t|^{-2.5}$ : it diverges rather rapidly as the critical point is approached. For $m$ not conserved, $z$ is close to 2 and so putting in the values for $\eta$ and $\nu$ we have that $\tau_{\xi}$ scales as $|t|^{-1.3}$ : it still diverges but more slowly. So, the closer the critical point is approached the longer is the waiting time before our expressions for the nucleation barrier, and size of the nucleus are valid. This, together with the effect of gravity on long wavelength fluctuations will limit how closely the critical point can be approached and the behaviour predicted by Eqs. (7) and (8) observed.

\section{NUCLEI WHICH DO NOT COUPLE TO THE ORDER PARAMETER AND NON-ISING UNIVERSALITY CLASSES}

Section III considered the case of a nucleus near an Ising-like transition, and in which the nucleus couples to 
the order parameter. In this section we start by considering nuclei near an Ising-like critical point which do not couple to the order parameter, and then move on to consider critical points in other universality classes. There are results for colloidal particles in fluids near Ising-like critical points where the particles do not couple to the order parameter [15]. This is so when the two phases which form below $T_{\beta}$ are related by symmetry and the nucleus does not discriminate between these two phases. Now, whether or not a nucleus (or colloidal particle) couples to the order parameter, it will in general couple to the energy density $u$. The perturbation to the energy density around a particle/nucleus is given by the energy correlation function. A scaling argument analogous to that in the previous section can be constructed to yield the result that the coupling to the energy density yields a singular part in the free-energy barrier which scales as the energy density $u$, i.e.,

$$
\Delta F_{S}^{*}=|t|^{1-\alpha} Z_{ \pm}\left(h /|t|^{\beta \delta}\right),
$$

where $\alpha$ is the specific-heat exponent, and $Z_{ \pm}$are scaling functions. The right-hand side of Eq. (19) is the appropriate scaling for the energy density. Now, $1-\alpha>\beta$, and so if a nucleus couples to both $m$ and $u$ then the singular term from the $m$ coupling, Eq. (13), will dominate and we can neglect the singularity of Eq. (19): we did this in the previous section. In general, we always expect the nucleus to couple to the energy density but the resulting singularity, Eq. (19) will be dominated by that from the $m$ coupling unless the latter is absent in which case Eq. (19) should give the dominant singularity in the free energy barrier to nucleation. Note that the temperature derivative of the nucleation barrier $\Delta F^{*} / T$. varies as the heat capacity, i.e., as $|t|^{-\alpha}$ at $h=0$.

So, for a nucleus which does not couple to the order parameter, we still have a singularity in the free-energy barrier, Eq. (19), but it is weak. In three dimensions, $\alpha=0.11$ and so the temperature derivative of the freeenergy barrier diverges only as $|t|^{-0.11}$ at $h=0$ : this weak divergence may be difficult to observe. Also, at $h=0$, if the nucleus does not couple to $m$, then $m^{*}=0$ at $h=0$ by symmetry. Thus, Eq. (11), the field derivative of $\partial \Delta F^{*} / \partial h=0$ at $h=0$.

As mentioned in the introduction, in fluid systems like protein solutions and liquid mixtures, there is no symmetry available to prevent the coupling of the nucleus to $m$. So the results of the previous section apply there, and the coupling to the energy density is subdominant. However, let us consider systems in universality classes other than that of the Ising model. We speculate that our finding of singular terms in the free-energy barrier to nucleation near a critical point is not restricted to systems with a scalar order parameter. Even if the order parameter is, say a two-dimensional unit vector as it is in the universality class of the XY-model [3, 4 , then the nucleus of a new phase should couple to the critical fluctuations. For example, the fluid to superfluid transition in liquid ${ }^{4} \mathrm{He}$ is in the XY universality class. Now, the vector order parameter is the phase of a wavefunction and a nucleus of another phase, e.g., the vapour phase, will not couple to the phase of a quantum mechanical wavefunction: $\Delta F^{*}$ does not depend on the local phase of the wavefunction. However, there is no reason for the nucleus not to couple to energy fluctuations near the fluid to superfluid transition. If it does so then $\Delta F^{*}$ will contain a singular term of the form of Eq. (19).

The fluid to superfluid transition crosses the liquidvapour transition [4], and so nucleation of the vapour phase can occur near the continuation of the superfluidfluid transition into conditions of pressure and temperature such that the vapour phase is the equilibrium phase. However, for the XY model in three dimensions, $\alpha$ is (very small but) negative which means the heat capacity, and thus the temperature derivative of $\Delta F^{*}$, has a cusp only: it does not diverge. This may make proving or disproving our speculation difficult.

\section{CONCLUSION}

We have considered the effect of a critical point on the nucleation of a new phase of another phase transition. The order parameter associated with the critical point is a scalar, putting the transition in the universality class of the Ising model. The critical point results in power-law singularities in both the free energy barrier to nucleation, $\Delta F^{*}$, and the size of the nucleus, $m^{*}$. The singular term in $\Delta F^{*}$ has the same scaling as the order parameter, and that in $m^{*}$ has the scaling of the response function of the order parameter. Thus, for example, as the critical point is approached at zero field, the singular term in $\Delta F^{*}$ varies as $|t|^{\beta}$, where $\beta$ is the usual critical exponent and $t$ is the temperature minus that at the critical point. As $\beta>0$, the singular term in the free energy barrier tends to zero as the critical point is approached. However, derivatives of $\Delta F^{*}$, both with respect to the temperature and to the external field, diverge as the critical point is approached: the free-energy barrier to nucleation varies rapidly near the critical point. These predictions are universal, they apply to nucleation in any system in which nucleation of a new phase occurs near a critical point in the universality class of the Ising model.

All the above holds given only that the core of the nucleus, where the nucleus resembles the new bulk phase that is nucleating, couples to the order parameter. It will do so unless prevented by a symmetry. This symmetry is clearly absent in fluid systems, in which the order parameter is a density and/or composition difference. In the previous section we speculated that there are also singular terms in $\Delta F^{*}$ near critical points in other universality classes. In other universality classes it is clear 
that symmetry can prevent coupling to the order parameter, in which case the singularity in $\Delta F^{*}$ will be weaker.

The relaxation time diverges as the critical point is approached, see section III. Thus, in experiment there is a limit to how close the critical point can be approached before the relaxation time becomes prohibitively long. This may limit the minimum value of $|t|$ that is achievable or it may be limited by some other factor. For example, the protein lysozyme has a fluid-fluid transition with a critical point within the fluid-crystal coexistence region [12], and thus is a candidate for studying nucleation of a non-critical phase, here a crystalline phase, near an Ising-like critical point. Far from the critical point the characteristic relaxation time can be estimated as the time a lysozyme molecules takes to diffuse its own diameter. The single particle diffusion constant of lysozyme $D=O\left(10^{-10} \mathrm{~m}^{2} \mathrm{~s}\right)$ [26], and its diameter is approximately $4 \mathrm{~nm}$ [26]. This gives a relaxation time of order $10^{-7} \mathrm{~s}$. Using this as the order of magnitude of the amplitudes $\tau_{ \pm}$, we have that at $h=0$, the relaxation time $\tau_{\xi}$ is of order $10^{-7}|t|^{-2.5}$ s at a temperature $t$. The exponent of $t$ is $z \nu$, with $z$ taking its value for a conserved order parameter. Now, assuming careful temperature control the critical temperature can be approached to within, say, $0.1^{\circ} \mathrm{C}$. As the effective interactions between the molecules in solution vary strongly with temperature $0.1^{\circ} \mathrm{C}$ should correspond to $|t|=O\left(10^{-2}-10^{-3}\right)$, not $|t|=4 \times 10^{-4}$ as it would for temperature independent interactions. At $|t|=10^{-3}$, we have a relaxation time $\tau_{\xi}=O(1 \mathrm{~s})$ : still rather short. Thus, in experiments on globular protein molecules like lysozyme, limitations on how well the temperature can be controlled are the limiting factor; the relaxation time $\tau_{\xi}$ is always well within the range accessible in experiment.

It is a pleasure to acknowledge discussions with $\mathrm{R}$. Evans, A. Parry and P. Upton. This work was supported by EPSRC (GR/N36981).

[1] Here we consider only homogeneous nucleation, nucleation in the bulk, far from any interface. Heterogeneous nucleation, nucleation at an interface, either with a wall or an impurity, is in fact more common, and is not always an activated process.
[2] P. G. Debenedetti, Metastable Liquids (Princeton University Press, Princeton, 1996).

[3] L. P. Kadanoff, Statistical Physics (World Scientific, Singapore, 2000).

[4] P. M. Chaikin and T. C. Lubensky, Principles of Condensed Matter Physics (Cambridge University Press, Cambridge, 1995).

[5] R. P. Sear, J. Chem. Phys. 114, 3170 (2001).

[6] R. P. Sear, Phys. Rev. E 63, 066105 (2001).

[7] V. Talanquer and D. W. Oxtoby, J. Chem. Phys. 109, 223 (1998).

[8] P. R. ten Wolde and D. Frenkel, Science 277, 1975 (1997).

[9] N. M. Dixit and C. F. Zukoski, J. Coll. Int. Sci. 228, 359 (2000).

[10] R. Piazza, Curr. Opinion Coll. Int. Sci. 5, 38 (2000).

[11] M. L. Broide, C. R. Berland, J. Pande, O. O. Ogun and G. B. Benedek, Proc. Nat. Acad. Sci. 88, 5660 (1991).

[12] M. Muschol and F. Rosenberger, J. Chem. Phys. 107, 1953 (1997).

[13] S. D. Durbin and G. Feher, Ann. Rev, Phys. Chem. 47, 171 (1996).

[14] P. G. de Gennes, C. R. Acad. Sci Paris II 292, 701 (1981).

[15] T. W. Burkhardt and E. Eisenriegler, Phys. Rev. Lett. 74, 3189 (1995).

[16] E. Eisenriegler and U. Ritschel, Phys. Rev. B 51, 13717 (1995).

[17] A. Hanke and S. Dietrich, Phys. Rev. E 59, 5081 (1999).

[18] Classical nucleation theory predicts that for reasonable values of the interfacial tension (here that between the $\alpha_{H T}$ and $\alpha_{L T}$ phases) the $\Delta F^{*}$ will be of the right order for nucleation to occur when the nucleus contains $O(10)$ spins. As critical fluctuations change $\Delta F^{*}$ by an amount $O(k T)$, this conclusion still holds near a critical point.

[19] See Ref. 24 for a careful, thermodynamic, derivation of Eq. (11). It is Eq. (24) of this reference. Note that they use language appropriate to fluids, so our $m^{*}$ is their $\Delta N$ the excess number of molecules in the nucleus and the field is not $h$ but $\mu$ the chemical potential of the molecules.

[20] R. Guida and J. Zinn-Justin, J. Phys. A 31, 8103 (1998).

[21] D. Kashchiev, J. Chem. Phys. 76, 5098 (1982).

[22] Y. Viisanen, R. Strey and H. Reiss, J. Chem. Phys. 99, 4680 (1993).

[23] R. K. Bowles, R. McGraw, P. Schaaf, B. Senger, J.-C. Voegel and H. Reiss, J. Chem. Phys. 113, 4524 (2000).

[24] R. K. Bowles, D. Reguera, Y. Djikaev and H. Reiss, J. Chem. Phys. 115, 1853 (2001).

[25] P. C. Hohenberg and B. I. Halperin, Rev. Mod. Phys. 49, 435 (1977).

[26] M. Muschol and F. Rosenberger, J. Chem. Phys. 103, 10424 (1995). 


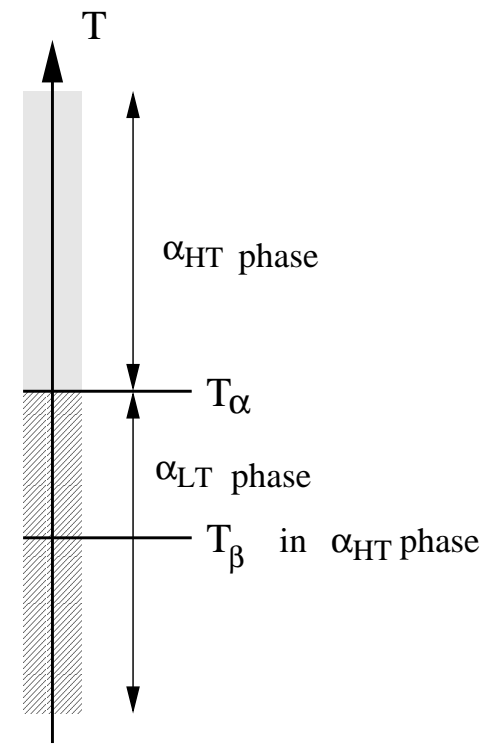

FIG. 1. Schematic phase behaviour along the temperature axis, at $h=0$. The shaded (hatched) region denotes the temperature range over which the high (low) temperature phase of transition $\alpha$ is the equilibrium phase. The temperatures of the two transitions are labelled by $T_{\alpha}$ and $T_{\beta}$.

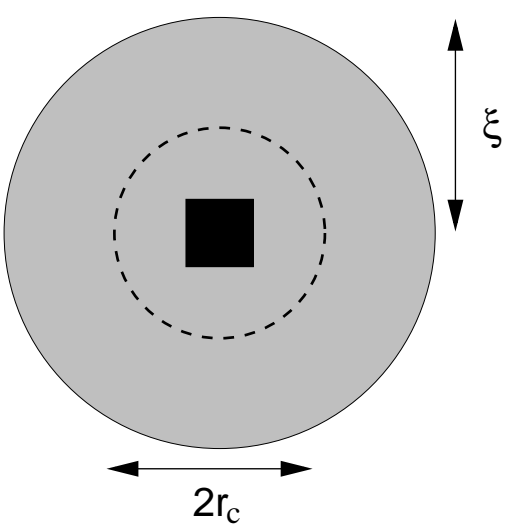

FIG. 2. Schematic of a nucleus of the ordered phase of transition $\alpha$ near transition $\beta$. The core of the ordered phase of transition $\alpha$ is solid black, and the perturbation this causes in the surroundings is the shaded circle of radius the correlation length $\xi$. The spherical boundary with radius $r_{c}$ is denoted by the dashed circle. 